\title{
ZERO DISTRIBUTION FOR PAIRS OF HOLOMORPHIC FUNCTIONS WITH APPLICATIONS TO EIGENVALUE DISTRIBUTION \\ BY
}

A. A. SHKALIKOV

\begin{abstract}
ABSTRACr. Let $f$ and $g$ be holomorphic in an angle $\Lambda$. Theorem 1 shows that the zero-distributions of $f$ and $g$ are comparable if, near $\partial \Lambda, f$ and $g$ grow similarly. This result is applied to analyse the asymptotic behavior of eigenvalues of certain perturbed normal operators.
\end{abstract}

1. Introduction. For $\frac{1}{2}<\alpha<\infty$ let

$$
\Lambda=\Lambda_{\alpha}=\{z ;|\arg z| \leqslant \pi / 2 \alpha\},
$$

and let $f$ and $g$ be holomorphic in (the closure of) $\Lambda_{\alpha}$. If $\Lambda$ were bounded, Roché's theorem would guarantee that $f$ and $g$ have the same number of zeros in $\Lambda$ if, say, $|f(z)-g(z)|<g(z)$ for $z \in \partial \Lambda$. Our main result provides a way of obtaining similar conclusions for $\Lambda$ as defined in (1.1); the method is to compare the behavior of $f$ and $g$ near $\partial \Lambda$ to that of an appropriate regularly varying auxiliary function.

The auxiliary functions considered will be nonnegative, nondecreasing functions defined for $t>0$, with their behavior limited as $t \rightarrow \infty$. If $\phi(t)$ and $\psi(t)$ are nonnegative and nondecreasing for $t>0$, we say

$$
\phi \curvearrowleft \psi
$$

if $|\psi(t) / \phi(t)|^{ \pm 1}=O(1)(t \rightarrow \infty)$, and

$$
\phi \sim \psi
$$

if $\phi(t)=\{1+o(1)\} \psi(t)(t \rightarrow \infty)$.

For a fixed $p>0$, the class $A(p)$ consists of nonnegative, nondecreasing functions $\phi(t)(t>0)$ such that there exists $a>1$ such that

$$
\phi(a t) \leqslant a^{p} \phi(t) \quad\left(t>t_{0}(a)\right),
$$

and we set

$$
A=\bigcup A(p) \quad(p>0)
$$

in (1.4) and below, the qualification $t>t_{0}(\alpha, \beta, \ldots)$ means: when $t$ is sufficiently large; this bound may depend on the parameters $\alpha, \beta, \ldots$, and the choice of $t_{0}$ is not

Received by the editors April 30, 1980 and, in revised form, February 12, 1981.

1980 Mathematics Subject Classification. Primary 30D20; Secondary 47A70.

Key words and phrases. Zero distribution of holomorphic functions, estimates of Blaschke product, eigenvalue distribution of perturbed normal operators.

(C)1984 American Mathematical Society $0002-9947 / 84 \$ 1.00+\$ .25$ per page 
necessarily the same at each occurence. Condition (1.4) implies that $\phi$ has no Pólya-peaks of order $>p[7$, p. 101] and an immediate consequence of (1.4) is that

$$
\phi(y) \leqslant C(y / t)^{p} \phi(t) \quad\left(t_{0} \leqslant t \leqslant y, C=C(a)\right) .
$$

The class $B$ consists of functions $\phi(t)(t>0)$ which are nonnegative, nondecreasing, such that for some $a>1$,

$$
\phi(a t) \geqslant 2 \phi(t) \quad\left(t>t_{0}(a)\right) .
$$

Finally, if given $\varepsilon>0$ we can find $\delta>0$ such that

$$
\phi((1+\delta) t) \leqslant(1+\varepsilon) \phi(t) \quad\left(t>t_{0}\right),
$$

then we say $\phi \in C$. Clearly, $C \subset A$.

In order to state our main result, we first recall that if $f$ is analytic in the sector $\Lambda=\Lambda_{\alpha}$ of (1.1), and if $M(r, f, \Lambda)=\max |f(z)|(|z|=r, z \in \Lambda)$, then the order $\rho$ of $f$ is

$$
\rho=\limsup _{r \rightarrow \infty} \frac{\log \log M(r, f, \Lambda)}{\log r} .
$$

The zeros of $f$ are counted by the usual functionals

$$
n_{f}(r)=\sum_{\substack{\left|z_{k}\right|<r \\ z_{k} \in \bar{\Lambda}}} 1, \quad N_{f}(r)=\int_{0}^{r} n_{f}(t) t^{-1} d t
$$

where the $z_{k}$ are the roots of $f$ in the interior of $\Lambda$. We assume throughout that $n_{f}(r)$ and $n_{g}(r)$ are zero for $0 \leqslant r<h$ for some fixed $h>0$.

THEOREM 1. Let $f$ and $g$ be holomorphic of order $\leqslant \alpha$ in the sector $\Lambda_{\alpha}$, such that all their zeros are contained in $\Lambda_{\alpha+\tau}$ for some $\tau>0$. Suppose the zeros of $g$ are regularly distributed in the sense that $n_{g}(r) \in B \cap A(p)(c f .(1.3),(1.4))$ for some $p<\alpha$, and that

$$
|\ln | f(z) / g(z)||=o\left(n_{g}(r)\right)
$$

uniformly as $z \rightarrow \infty$ in $\Lambda_{\alpha}-\Lambda_{\alpha+\tau}$. Then, using the notation (1.2),

$$
N_{g}(r) \asymp N_{f}(r), \quad n_{g}(r) \asymp n_{f}(r) .
$$

Note 1. In Theorem 2 , in $\$ 4$, we shall modify Theorem 1 to obtain conditions needed to deduce that $N_{g} \sim N_{f}$ and $n_{g} \sim n_{f}$.

Note 2. In general, equivalence of $N_{f}$ and $N_{g}$ is not equivalent to that of $n_{f}$ and $n_{g}$; this is where the assumption $n_{g} \in B \cap A(p)$ is needed. We remark that if $n(t) \in B$, then

$$
N(r) \equiv \int_{0}^{r} n(t) t^{-1} d t \leqslant C n(r)
$$

and if $n(t) \in A$ then

$$
n(r) \leqslant C N(r) .
$$


We sketch the proofs. If $n \in B$, take $a>1$ so that (1.7) holds. For $r>t_{0}$, choose $j$ with $a^{j} t_{0} \leqslant r \leqslant a^{j+1} t_{0}$; then

$$
\begin{aligned}
N(r) & \leqslant \int_{0}^{t_{0}} n(t) t^{-1} d t+\sum \int_{a^{h} t_{0}}^{a^{h+1} t_{0}} n(t) t^{-1} d t \\
& =O(1)+O(\log a) n\left(a^{j+1} t_{0}\right) \leqslant C n(r) .
\end{aligned}
$$

The proof of (1.3) is easier, using (1.6):

$$
N(r) \geqslant \int_{r / 2}^{r} n(t) t^{-1} d t \geqslant C n\left(\frac{r}{2}\right) \int_{r / 2}^{r}\left(\frac{r}{t}\right)^{p} t^{-1} d t \geqslant C n(r) .
$$

2. Preliminary remarks and lemmas. By replacing $z$ by $z^{2(2 \alpha+\tau)^{-1}}$, we may assume $\alpha=1-\delta$, with $\delta=\tau(2 \alpha+\tau)^{-1}$, and that (1.10) holds in $\Lambda_{1-\delta}-\Lambda_{1+\delta}$. The hypothesis of Theorem 1 now is that $n_{g}(r) \in B \cap A\left(p^{\prime}\right)$, where $p^{\prime}=2 p(2 \alpha+\tau)^{-1}$ $<1$ and that both $f$ and $g$ have order $\leqslant 1-\delta$ in $\Lambda_{1-\delta}$. This normalization will be in effect through $\$ 4$.

Lemma 1. Let $\delta>0$ be as just described. Then, given $\varepsilon>0$, we have

$$
n_{f}(r)+n_{g}(r) \leqslant r^{1-\delta+\varepsilon} \quad\left(r>r_{0}\right) .
$$

Proof. The function $f_{1}(z)=f\left(z^{1 /(1-\delta)}\right)$ is holomorphic in $\Lambda_{1}$, with zeros in $\Lambda_{1+8^{\prime}}$ (where $\delta^{\prime}=2 \delta(1-\delta)^{-1}$ ) and $f_{1}$ has order $\leqslant 1$. Since order $\left(f_{1}\right) \leqslant 1$, we obtain from [9, Theorem 4, Chapter 4] that $\nu_{f_{1}}(r) \leqslant r^{1+\varepsilon / 2}\left(r>r_{0}\right)$, where $\nu_{f_{1}}(r)$ is the number of zeros of $f_{1}$ in $\{|z-r / 2|<r / 2\}$. But all zeros of $f_{1}$ are in $\Lambda_{1+\delta^{\prime}}$, so $n_{f_{1}}(r) \leqslant C \nu_{f_{1}}(r)$ $\leqslant r^{1+\varepsilon}\left(r>r_{0}\right)$, and since $n_{f}(r) \leqslant\left(n_{f_{1}}(r)\right)^{(1-\delta)}$, we have estimate (2.1) for $n_{f}(r)$. Similarly, $n_{g}(r)=O\left(r^{1-\delta+\epsilon}\right)(r \rightarrow \infty)$, and (2.1) follows.

COROLlary. Let $z_{k}$ be the zeros of $f$. Then the Blaschke product with zero set $z_{k}$ converges in the plane

$$
B(z)=B_{f}(z)=\prod\left(z_{k}-z\right) /\left(z_{k}+z\right) .
$$

The Blaschke product made from the zeros of $g, B_{g}(z)$, also converges. Both products represent bounded analytic functions in $\Lambda_{1}$ and meromorphic functions in the plane.

Proof. Lemma 1 implies that the Blaschke condition $\Sigma\left(\operatorname{Re} z_{k}\right) /\left(1+\left|z_{k}\right|^{2}\right)<\infty$ holds.

We can estimate the Nevanlinna characteristic [7] of $B_{g}$; only later, in Lemma 9, we can obtain an equivalent estimate for $B_{f}$.

LEMMA 2. The meromorphic function $B_{g}$ satsifies

$$
T\left(r, B_{g}\right) \leqslant C n_{g}(r)=O\left(r^{1-\delta+\varepsilon}\right) .
$$

Proof. Let $z_{k}$ be the zeros of $g$ in $\Lambda_{1+\delta}$ and

$$
P_{1}(z)=\prod\left(1-z / z_{k}\right), \quad P_{2}(z)=\prod\left(1+z / z_{k}\right) ;
$$

Lemma 1 implies that both products converge to a function of order $<1$. We can say a little more: since $n\left(r, P_{i}\right)=n_{g}(r) \in A_{p}(i=1,2)$, the usual estimate for a 
canonical product [7, p. 27, formula (1.21) with $q=1]$ and (1.6) show

$$
\begin{aligned}
\ln \left|P_{i}(z)\right| & \leqslant \int_{0}^{r} \frac{n_{g}(t)}{t} d t+r \int_{r}^{\infty} \frac{n_{g}(t)}{t^{2}} d t \\
& \leqslant N_{g}(r)+C r^{1-p} n_{g}(r) \int_{r}^{\infty} t^{p-2} d t \leqslant C n_{g}(r) .
\end{aligned}
$$

Since $B_{g}=P_{1} P_{2}^{-1}$, Nevanlinna's first fundamental theorem implies that

$$
T\left(r, B_{g}\right) \leqslant T\left(r, P_{1}\right)+T\left(r, P_{2}\right)+O(1) \leqslant C n_{g}(r),
$$

which, with Lemma 1, is (2.3).

COROLlaRY. The characteristic of the Blaschke product $B_{f}$ satisfies

$$
T\left(r, B_{f}\right)=O\left(r^{1-\delta+\varepsilon}\right) .
$$

Remark. Theorem 1 implies the more refined result that (2.3) holds with $B_{f}$ in place of $B_{g}$.

Proof. Define $P_{1}$ and $P_{2}$ as in the proof of Lemma 2, now with $z_{k}$ the zeros of $f$. Estimate (2.1) on $n_{f}(t)$ may be used to estimate $T\left(r, P_{1}\right)$ and $T\left(r, P_{2}\right)$ as was done above. We thus obtain $T\left(r, B_{f}\right) \leqslant T\left(r, P_{1}\right)+T\left(r, P_{2}\right)+O(1)=O\left(r^{1-\delta+\varepsilon}\right)$, which is (2.4).

Lemma 3. Let $F(z)$ be holomorphic in $\Lambda_{1}$, with all zeros in $\Lambda_{1+\delta}$ for some $\delta>0$. Then

$$
\begin{aligned}
N_{F}(r)= & \frac{1+\delta}{\pi^{2} \delta} \int_{0}^{r}\left[J_{F}\left(\frac{\pi}{2}, t\right)-J_{F}\left(\frac{\pi}{2(1+\delta)}, t\right)\right. \\
& \left.-J_{F}\left(-\frac{\pi}{2}, t\right)+J_{F}\left(-\frac{\pi}{2(1+\delta)}, t\right)\right] \frac{d t}{t} \\
+ & \frac{2(1+\delta)^{2}}{\pi^{3} \delta^{2}} \int_{\pi / 2(1+\delta)}^{\pi / 2} \int_{-\pi / 2}^{-\pi / 2(1+\delta)} \int_{\psi}^{\theta} \ln \left|F\left(r e^{i \phi}\right)\right| d \phi d \psi d \theta
\end{aligned}
$$

where

$$
J_{F}(\phi, t)=\int_{0}^{t} \ln \left|F\left(s e^{i \phi}\right)\right| s^{-1} d s .
$$

Proof. This follows from the generalized Jensen formula as presented in [9, p. 143]. Let $n_{F}(t, \psi, \theta)$ be the number of zeros of $F$ inside $S(t, \psi, \theta) \equiv\{\psi<\arg z<\theta\}$ $\cap\{|z|<t\}$, and $N_{F}(r, \psi, \theta)=\int_{0}^{r} n_{F}(t, \psi, \theta) t^{-1} d t$.

If $F \neq 0$ on $\partial S(t, \psi, \theta)$, then the generalized Jensen formula implies that

$$
\begin{aligned}
N_{F}(r, \psi, \theta)= & \frac{1}{2 \pi}\left[\frac{d}{d \phi} \int_{0}^{r} J_{F}(\phi, t) t^{-1} d t\right]_{\phi=\theta} \\
& -\frac{1}{2 \pi}\left[\frac{d}{d \phi} \int_{0}^{r} J_{F}(\phi, t) t^{-1} d t\right]_{\phi=\psi}+\frac{1}{2 \pi} \int_{\psi}^{\theta} \ln \left|F\left(r e^{i \phi}\right)\right| d \phi .
\end{aligned}
$$

Lemma 3 follows from (2.5) on integrating with respect to $\psi$ and $\theta$, and recalling that all zeros of $F$ are in $\Lambda_{1+\delta}$. 
We may use Lemma 3 to give a representation for $N_{f}(r)-N_{g}(r)$. Let $B_{f}$ and $B_{g}$ be the Blaschke products made from the zeros of $f$ and $g$, and

$$
B^{*}(z)=B_{f}(z) / B_{g}(z),
$$

so that $B^{*}$ is meromorphic (Corollary to Lemma 1). Since $\left|B^{*}(i y)\right|=1$, we obtain from Lemma 3:

LEMMA 4. The counting functions of $f$ and $g$ are related by

$$
\begin{aligned}
N_{f}(r)-N_{g}(r)= & \frac{1+\delta}{\pi^{2} \delta} \int_{0}^{r}\left[I\left(\frac{\pi}{2(1+\delta)}, t\right)-I\left(-\frac{\pi}{2(1+\delta)}, t\right)\right] t^{-1} d t \\
& +\frac{2(1+\delta)^{2}}{\pi^{3} \delta^{2}} \int_{\pi / 2(1+\delta)}^{\pi / 2} \int_{-\pi / 2}^{-\pi / 2(1+\delta)} \int_{\psi}^{\theta} \ln \left|B^{*}\left(r e^{i \phi}\right)\right| d \phi d \psi d \theta,
\end{aligned}
$$

where

$$
I(\phi, t)=\int_{0}^{t} \ln \left|B^{*}\left(s e^{i \phi}\right)\right| s^{-1} d s .
$$

The proof of Theorem 1 will follow on estimating the terms on the right side of (2.7). This will be done in $\$ 3$. Our final results here seem to be new, although Lemma 6 has also been obtained by G. V. Radzievskii (unpublished).

Lemma 5. Let $F$ be holomorphic in $\Lambda_{\alpha}$, of order $<\alpha$, and suppose

$$
\ln |F(z)| \leqslant \phi(r) \quad\left(z \in \partial \Lambda_{\alpha}\right)
$$

where $\phi \in A(p)$ for some $p<\alpha(c f$. (1.4)). Then

$$
\ln |F(z)| \leqslant C \phi(|z|) \quad\left(z \in \Lambda_{\alpha}\right)
$$

for some constant $C$.

Proof. This lemma is similar to a result of Beurling [3, p. 34]. However, we assume that $\phi$ has order strictly less than $\alpha$, and satisfies (1.4), but in turn deduce the global conclusion (2.9).

Consider $\Phi(z)=F(z) e^{-\varepsilon z^{p}-b}$ in $\Lambda_{\alpha}$, where we take $x^{p}>0$ if $x>0$. The constants $b$ and $\varepsilon$ are determined as follows. Let $t_{0}$ be associated to $f$ by (1.4), let $r>t_{0}$; we take $b=f(r)$ and $\varepsilon=a^{p} f(r) r^{-p} \cos ^{-1}(\pi p /(2 \alpha))$. It is routine to see that

$$
f(t)-\varepsilon t^{p} \cos (\pi p / 2 \alpha)-b \leqslant 0 .
$$

This is clear when $t \leqslant r$, for $f$ is nondecreasing. If $t>r$, say $a^{j} r \leqslant t \leqslant a^{j+1} r$ $(j=1,2, \ldots)$ then $(1.4)$ gives

$$
f(t) \leqslant a^{(j+1) p} f(r) \leqslant a^{p}(t / r)^{p} f(r)=\varepsilon t^{p} \cos (\pi p /(2 \alpha)),
$$

and (2.10) holds. Now (2.10) implies

$$
\ln \left|\Phi\left(t e^{ \pm i \pi / 2 \alpha}\right)\right| \leqslant f(t)-\varepsilon t^{p} \cos (\pi p / 2 \alpha)-b \leqslant 0,
$$

and the Phragmén-Lindelöf principle shows that $\ln |\Phi(z)| \leqslant 0\left(z \in \Lambda_{\alpha}\right)$. This gives (2.9) with $C=a^{p} \cos ^{-1}(\pi p / 2 \alpha)+1$. 
COROLlaRY. Let $F$ be holomorphic in $\Lambda_{\alpha}$, of order $<\alpha$, and suppose

$$
\ln \left|F\left(r e^{ \pm i \pi / 2 \alpha}\right)\right|=o(\phi(r)) \quad(r \rightarrow \infty)
$$

where $\phi \in A(p)$ for some $p<\alpha$. Then

$$
\ln |F(z)|=o(\phi(|z|)) \quad\left(z \rightarrow \infty, z \in \Lambda_{\alpha}\right) .
$$

LEMMA 6. Let $F(z)$ be holomorphic, of order $\leqslant \rho$ in $\Lambda_{\alpha}$, and have no zeros in $\Lambda_{\alpha}$. Then for any $\varepsilon>0$, the function $F^{-1}(z)$ has order $\leqslant \rho^{*} \equiv \max (\rho, \alpha)$ in $\Lambda_{\alpha+\varepsilon}$.

Proof. Let $f(\lambda)$ be a holomorphic nonzero in $\Lambda_{1}$ and $|f(\lambda)|<M$. Consider the function $g(\lambda)=\ln M-\ln f(\lambda)$. Since $\operatorname{Re} g(\lambda)>0$ as $\lambda \in \Lambda_{1}$, we may apply Carathéodory's inequality (see [9, Chapter 1, \$7])

$$
g(\lambda)<5|g(1)| r / \sin \theta, \quad \lambda=r e^{i \theta},|\lambda|>1 .
$$

This implies

$$
\ln \left|M f^{-1}(\lambda)\right| \leqslant\left|-\ln M f^{-1}(\lambda)\right|=\left|\ln M f^{-1}(\lambda)\right| \leqslant C r / \sin \theta
$$

and hence

$$
\ln \left|f^{-1}(\lambda)\right| \leqslant C r, \quad \lambda \in \Lambda_{1+\varepsilon}, \varepsilon>0 .
$$

Suppose $\alpha \geqslant \rho$. Take any $\varepsilon>0$ and consider the function $G(z)=F(z) \exp \left(-z^{\alpha+\varepsilon}\right)$, which is bounded in $\Lambda_{\alpha+2 \varepsilon}$. Then the function $f(\lambda)=G\left(\lambda^{1 /(\alpha+2 \varepsilon)}\right)$ satisfies (2.11) and we have

$$
\ln \left|F^{-1}(z)\right| \leqslant C|z|^{\alpha+2 \varepsilon}, \quad z \in \Lambda_{\alpha+3 \varepsilon} .
$$

Now let $\alpha<\rho$. We have from (2.12),

$$
\ln \left|F^{-1}(z)\right| \leqslant C|z|^{\rho+\varepsilon}, \quad z \in \Lambda_{\rho+\varepsilon} .
$$

But we may obtain (2.13) in any sector $\Lambda_{\rho+\varepsilon}^{\phi}=\{\lambda:|\phi-\arg \lambda| \leqslant \pi / 2(\rho+\varepsilon)\}$ if $\Lambda_{\rho+\varepsilon}^{\phi} \subset \Lambda_{\alpha+\varepsilon}$, hence estimate (2.13) is valid, as $z \in \Lambda_{\alpha+\varepsilon}$. This proves Lemma 2 with (2.12).

\section{Estimates for Theorem 1.}

LEMMA 7. The quotient $B^{*}(z)$ satisfies

$$
|\ln | B^{*}(z)||=o\left(n_{g}(r)\right)
$$

as $|z| \rightarrow \infty$ in $\Lambda_{1}-\Lambda_{1+\delta}$.

Proof. Let

$$
\chi_{1}(z)=f(z) B_{f}(z)^{-1}, \quad \chi_{2}(z)=g(z) B_{g}(z)^{-1}, \quad \Delta(z)=\chi_{1}(z) \chi_{2}(z)^{-1} .
$$

We claim that if $\rho^{*}$ is the order of $\Delta$ in $\Lambda_{1}$ then

$$
\rho^{*}<1 \text {. }
$$


Once (3.2) is established, it is clear how to get (3.1). For since $\left|B_{f}(i y)\right|=\left|B_{g}(i y)\right| \equiv 1$ $(-\infty<y<\infty)$, we see from $(1.10)$ that

$$
|\ln | \Delta(i y)|| \equiv|\ln | \frac{f(i y)}{g(i y)}||=o\left(n_{g}(y)\right) \quad(-\infty<y<\infty),
$$

so (3.2) allows us to use the corollary to Lemma 5 with $\alpha=1$, and deduce that

$$
|\ln | \Delta(z)||=o\left(n_{g}(r)\right)
$$

as $|z|=r \rightarrow \infty$ in $\Delta_{1}$. But now this and (1.10) give the lemma since

$$
\pm \ln \left|B^{*}(z)\right|=\mp|\Delta(z)| \pm \ln |f(z) / g(z)| \text {. }
$$

We now prove (3.2). The functions $\chi_{i}$ are holomorphic and nonzero in $\Lambda_{1-\delta}$. We claim that the order $\rho_{i}$ of $\chi_{i}$ is $\leqslant 1-\delta$ in $\Lambda_{1-\delta}$; once this is known, (3.2) is a consequence of Lemma 6 with $\rho=1-\delta$ and $\alpha=1-\delta$ (so that $\varepsilon=\delta$ ). Let us consider $\chi_{1}$ in the region $D(R)=\Lambda_{1-\delta} \cap\{|z| \leqslant R\}$ for certain large $R$. On arg $z=$ $\pm \pi / 2(1-\delta)$, we recall that $f$ has order $1-\delta$ and $\left|B_{f}\right| \geqslant 1$, and hence

$$
\left|\chi_{1}(z) \exp \left(-2 z^{(1-\delta+\varepsilon)}\right)\right|=O(1)
$$

for any fixed $\varepsilon>0$. We next obtain a similar estimate on $\Lambda_{1-\delta} \cap\{|z|=R\}$ for an unbounded set of $R$. Since $T\left(r, B_{f}\right)=O\left(r^{1-\delta+\varepsilon}\right)$ (see (2.4)) we have from the $\cos \pi \rho$-theorem [10, p. 275] that

$$
\inf _{|z|=R} \ln \left|B_{f}(z)\right| \geqslant-R^{1-\delta+\varepsilon},
$$

for a sequence $R=R_{n} \rightarrow \infty$. Also, $f$ has order $\leqslant 1-\delta$ in $\Lambda_{1-\delta}$, so (3.3) holds on $\Lambda_{1-\delta} \cap\{|z|=R\}$ for these $R$. The maximum principle now gives (3.3) in all of $\Lambda_{1-\delta}$, so $\chi_{1}$ has order $\leqslant 1-\delta$ in $\Lambda_{1-\delta}$.

The same holds for $\chi_{2}$, and according to Lemma $6, \chi_{2}^{-1}$ has order less than $1-\delta$ in $\Lambda_{1-\delta}$. This establishes (3.2) and completes the proof of Lemma 7.

We now can make a major step toward Theorem 1.

LEMMA 8. We have $N_{f}(r) \leqslant C N_{g}(r), n_{f}(r) \leqslant C n_{g}(r)$.

Proof. Recall the definition of $I(\phi, t)$ from this statement of Lemma 4. Then Lemmas 6 and 7 give

$$
\int_{0}^{r} I\left( \pm \frac{\pi}{2(1+\delta)}, t\right) t^{-1} d t=o\left(n_{g}(r)\right)
$$

We next must estimate $\int_{\psi}^{\theta} \ln \left|B^{*}\left(r e^{i \phi}\right)\right| d \phi$ from above. Since $\left|B_{f}\right| \leqslant 1$ in $\Lambda_{1}$, it suffices to obtain upper bounds for $-\int_{\psi}^{\theta} \ln \left|B_{g}\left(r e^{i \phi}\right)\right| d \phi$; this is fortunate since Lemma 2 gives good information on $T\left(r, B_{g}\right)$. A useful lemma of Edrei and Fuchs [5, p. 322], (2.3) and (1.6) yield that if $I$ is a $\theta$-interval of length $|I|$, then

$$
\begin{aligned}
\int_{I}|\ln | B_{g}\left(r e^{i \phi}\right)|| d \phi & \leqslant \frac{25 R}{R-r} T\left(R, B_{g}\right)|I|\left\{1+\log ^{+} \frac{1}{|I|}\right\} \\
& \leqslant C n_{g}(r)|I|\left\{1+\log ^{+}|I|^{-1}\right\} \leqslant C n_{g}(r)
\end{aligned}
$$


if $R=2 r>r_{0}$. Estimates (3.4) and (3.5) may be inserted to (2.7), leading to

$$
N_{f}(r) \leqslant N_{g}(r)+o\left(n_{g}(r)\right)+C n_{g}(r)
$$

and an appeal to (1.12) and (1.13) gives

$$
N_{f}(r) \leqslant C N_{g}(r), \quad n_{f}(r) \leqslant C n_{g}(r) .
$$

It remains to reverse the inequalities of (3.6). We first improve the estimate of $T\left(r, B_{f}\right)$ from (2.4).

LEMMA 9. The characteristic of $B_{f}$ satisfies

$$
T\left(r, B_{f}\right)=O\left(n_{g}(r)\right) .
$$

Further if $r_{0}<r<R / 2$, we have

$$
T\left(r, B_{f}\right) \leqslant C N_{f}(R)+C(r / R)^{1-p} N_{g}(r),
$$

where $p<1$ is the class of $A(p)$ to which $n_{g}(r)$ belongs.

Proof. Write $B_{f}=P_{1}(f) P_{2}(f)^{-1}$ as in the proof of Lemma 2, where now the $z_{k}$ are the zeros of $f$. The information in (3.6) allows the estimates made for $T\left(r, P_{i}\right)$ $(i=1,2)$ in Lemma 2 to be transferred to $T\left(r, P_{1}(z)\right)$ and $T\left(r, P_{2}(z)\right)$. Thus $T\left(r, P_{i}(z)\right)=O\left(n_{g}(r)\right)$, and (3.7) follows.

An approximation lemma of Edrei and Fuchs [4, p. 296, with $q=0$ ] shows that

$$
\ln \left|P_{1}(z)\right|=\log \prod_{\left|z_{k}\right| \leqslant R}\left|1-\frac{z}{z_{k}}\right|+S(z, R) \quad\left(|z|=r<\frac{1}{2} R\right)
$$

where the remainder $S$ satisfies

$$
|S(z, R)| \leqslant 14(r / R) T\left(2 R, P_{1}\right) .
$$

Let us once more compute

$$
T\left(r, P_{1}\right)=\frac{1}{2 \pi} \int_{0}^{2 \pi} \ln ^{+}\left|P_{1}\left(r e^{i \theta}\right)\right| d \theta .
$$

Once again, as in [7, p. 27] (cf. our proof of Lemma 2) we have

$$
\int_{0}^{2 \pi} \sum_{\left|z_{k}\right| \leqslant R} \ln ^{+}\left|1-z_{k}^{-1} r e^{i \theta}\right| d \theta=O\left(N_{f}(R)\right),
$$

and since (3.7) implies that $T\left(r, P_{1}\right)=O\left(N_{g}(r)\right)$, (1.6) and (3.10) show that

$$
|S(z, R)| \leqslant C(r / R)^{1-p} N_{g}(r) \quad \text { if } R>2 r .
$$

This proves (3.8).

LEMMA 10. We have $N_{g}(r) \leqslant C N_{f}(r)$.

Proof. Since $\ln \left|B_{g}(z)\right| \leqslant 0$ for $z \in \Lambda_{1}$, (3.4) and (2.7) show

$$
N_{g}(r) \leqslant N_{f}(r)+\varepsilon n_{g}(r)+C \max _{-\pi / 2 \leqslant \psi<\theta \leqslant \pi / 2}\left[-\int_{\psi}^{\theta} \ln \left|B_{f}\left(f e^{i \phi}\right)\right| d \phi\right] .
$$


According to the Edrei-Fuchs lemma [3, p. 322] which was already used in (3.5), and our refined estimate (3.8) of $T\left(r, B_{f}\right)$, we see that

$$
\int_{\psi}^{\theta}|\ln | B_{f}\left(r e^{i \phi}\right)|| d \phi \leqslant C \frac{R}{R-r}\left\{N_{f}\left(R^{\prime}\right)+\left(\frac{R}{R^{\prime}}\right)^{1-p} N_{g}(R)\right\}
$$

uniformly in $-\pi / 2 \leqslant \psi<\theta \leqslant \pi / 2$, where $r \leqslant R / 2 \leqslant R^{\prime} / 4$. Given $\varepsilon>0$, take $R=$ $2 r$, and $R^{\prime}=c r$ so large that (uniformly in $\psi$ and $\theta$ )

$$
\int_{\psi}^{\theta}|\ln | B_{f}\left(r e^{i \phi}\right)|| d \phi \leqslant C N_{f}\left(R^{\prime}\right)+\varepsilon N_{g}(r)
$$

this is possible since $p<1, N_{g}(r)$ satisfies (1.6) and we know (3.7). Now (3.11) gives $(1-\varepsilon) N_{g}(r) \leqslant C N_{f}\left(R^{\prime}\right)$. But $N_{g}\left(R^{\prime}\right)=N_{g}(c r) \leqslant C N_{g}(r)$, hence $N_{g}\left(R^{\prime}\right) \leqslant C N\left(R^{\prime}\right)$ and Lemma 10 follows.

A combination of Lemmas 8, 10 and Note 2 gives $N_{f}(r) \asymp N_{g}(r) \asymp n_{g}(r)$. This implies that $N_{f}(r) \in A \cap B$, hence $n_{f}(r) \asymp N_{f}(r)$ and $n_{f}(r) \asymp n_{g}(r)$. Theorem 1 is proved.

4. Theorem on $\sim$ equivalence. The assumption of Theorem 1 does not allow us to deduce $N_{f} \sim N_{g}$ or $n_{f} \sim n_{g}$. As an example we consider the functions $f(z)=\sin z+$ $e^{\varepsilon z}, g(z)=\sin z$. We have $n_{g}(r) \in B \cap A_{p}, p=1$, and condition (1.10) holds uniformly, as $z \rightarrow \infty$ in $\Lambda_{1}-\Lambda_{\gamma}, 1<\gamma<(\pi / 2) \operatorname{arctg} \varepsilon$, but $n_{g}(r)=r / \pi+O(1)$, $n_{1}(r)=\sqrt{1+\varepsilon^{2}} r / \pi+O(1)$. The example of these functions shows that we can expect $n_{f} \sim n_{g}$ when the zeros of $f$ and $g$ asymptotically are contained in an arbitrary small sector and condition (1.10) holds outside of that sector.

THEOREM 2. Let $f$ and $g$ be holomorphic of finite order $\leqslant \beta$ in the sector $\Lambda_{\alpha_{0}}$, and for any $\alpha>\alpha_{0}$ all their zeros with the exception of a finite number are contained in $\Lambda_{\alpha}$. Suppose that

$$
|\ln | f(z) / g(z)||=O\left(n_{g}(r)\right)
$$

uniformly as $z \rightarrow \infty$ in $\Lambda_{\alpha_{0}}-\Lambda_{\alpha}$.

Then $N_{g}(r) \sim N_{f}(r)$, if $n_{g}(r) \in A \cap B$, and $n_{g}(r) \sim n_{f}(r)$ if $n_{g}(r) \in C \cap B$.

Proof. Since $n_{g}(r) \in A$ we can find $p$ such that $n_{g}(r) \in A_{p}$. If $\gamma=\max \left(\alpha_{0}, \beta p\right)$ and $\alpha>\gamma$, then (4.1) holds in $\Lambda_{\gamma}-\Lambda_{\alpha}$, and according to Theorem 1 we have

$$
n_{f}(r) \asymp n_{g}(r) \text {. }
$$

We may assume $\gamma<1$, otherwise we ought to replace $z$ by $z^{(\gamma+\tau)^{-1}}, \tau>0$.

As in Theorem 1 we have to estimate the terms in (2.7). According to (4.1) the first term on the right side of (2.7) has the estimate $o\left(N_{g}(r)\right)$. Using (3.5), (4.1) and (4.2), we see also that

$$
\begin{aligned}
& \int_{\theta}^{\psi} \ln \left|B^{*}\left(r e^{i \phi}\right)\right| d \phi=\left(\int_{-\varepsilon}^{\varepsilon}+\int_{\varepsilon}^{\theta}+\int_{\psi}^{-\varepsilon}\right) \ln \left|B^{*}\left(r e^{i \phi}\right)\right| d \phi \\
& \leqslant C \varepsilon\left(1+\log ^{+} \frac{1}{\varepsilon}\right) N_{g}(r)+\left(\int_{\varepsilon}^{\psi}+\int_{\theta}^{-\varepsilon}\right) \ln \left|B^{*}\left(r e^{i \phi}\right)\right| d \phi=o\left(N_{g}(r)\right) .
\end{aligned}
$$


Consequently, the second term on the right side of (2.7) has the estimate $o\left(N_{g}(r)\right)$, and according to definition (1.3) we have

$$
N_{g}(r) \sim N_{f}(r) \text {. }
$$

Now we deduce from (4.3) that $n_{f} \sim n_{g}$ if $n_{g}(r) \in B \cap C$.

Given $\varepsilon>0$ take $\delta>0$ such that

$$
n_{g}((1+\delta) r) \leqslant(1+\varepsilon) n_{g}(r)
$$

We have

$$
\begin{aligned}
{\left[n_{f}(r)\right.} & \left.-n_{g}((1+\delta) r)\right] \ln (1+\delta) \leqslant \int_{r}^{(1+\delta) r} \frac{n_{f}(t)-n_{g}(t)}{t} d t \\
& =N_{f}((1+\delta) r)-N_{g}((1+\delta) r)+N_{f}(r)-N_{g}(r) \\
& =o\left(N_{g}((1+\delta) r)\right)=o\left(n_{g}(r)\right) .
\end{aligned}
$$

Hence, from (4.4) we obtain

$$
n_{f}(r) \leqslant n_{g}((1+\delta) r)[1+o(1)] \leqslant(1+\varepsilon) n_{g}(r) .
$$

As in (4.5) we have

$$
\left[n_{g}(r)-n_{f}(r(1+\delta))\right] \ln (1+\delta) \leqslant \int_{r}^{(1+\delta) r} \frac{n_{g}(t)-n_{f}(t)}{t} d t=o\left(n_{g}(t)\right),
$$

Consequently,

$$
n_{f}(r(1+\delta)) \geqslant(1-\varepsilon) n_{g}(r(1+\delta)) .
$$

The combination of (4.6) and (4.7) gives Theorem 2.

5. Asymptotic behaviour of eigenvalues of certain perturbed normal operators. The first general result on the distribution of eigenvalues of an operator $(; H(I+S)$, where $H, S$ are compact operators and $H>0$, was established by M. V. Keldysh [8] (see also [6, Chapter V, §11]). He proved

$$
\sum_{\lambda_{k}^{-1}<r} 1=n_{I \prime}(r) \sim n_{G}(r)=\sum_{\left|u_{k}{ }^{1}\right|<r} 1,
$$

where $\lambda_{k}, \mu_{k}$ are the eigenvalues of operators $H$ and $(;$, respectively, provided that the function $n_{H}(r)$ satisfies some tauberian conditions.

This result has been generalized by many authors. The references can be found in [2] (see also [1]). As an application of Theorem 2 we will prove a result concerning this problem, which covers those of many authors.'

In this section we denote by $\sigma_{\infty}$ the collection of all compact operators and by $R$ the collection of all bounded operators acting in Hilbert space $\mathfrak{B}$. Also by $\lambda_{k}(A)$ we

\footnotetext{
'After submitting this paper for publication the author discovered a more complete and general result announced by A. S. Markus and V. I. Macaev in Functional Anal. Appl. (13 (1979), pp. 93-94 (Russian)). Using another method G. V. Radzievskii also obtained a result similar to Theorem 3 (Mat. Sb. 112 (1980), pp. 396-420 (Russian)).
} 
denote the eigenvalues of an operator $A$ and by $s_{k}(A)$ the $s$-numbers of this operator, i.e. the eigenvalues of $\left(A A^{*}\right)^{1 / 2}$. If $\sum s_{k}(A)<\infty\left(A \in \sigma_{1}\right)$, the characteristic determinant of the operator $A$ (see [6, Chapter $4, \S 1]$ ),

$$
\mathscr{D}_{A}(\mu)=\operatorname{det}(I-\mu A)=\prod_{k=1}^{\infty}\left(1-\mu \lambda_{k}(A)\right),
$$

converges. If $A(\mu)$ is a holomorphic operator-valued function in the domain $\Lambda \in \mathbf{C}$ with values in $\sigma_{1}$, then the function

$$
\operatorname{det}(I-A(\mu))=\prod_{k=1}^{\infty}\left(1-\lambda_{k}(A(\mu))\right)
$$

is holomorphic in the same domain [6, Chapter IV, $\S 1]$. Furthermore, if $\left(I-A\left(\mu_{0}\right)\right)^{-1}$ for at least one point $\mu_{0} \in \Lambda$, then from the results of $[3$, Chapter $1, \S 5]$ we conclude that $(I-A(\mu))^{-1} \in R$ for all $\mu \in \Lambda$ with the possible exception of certain isolated points $\left\{\mu_{k}\right\}$, and $\operatorname{dim} \mathcal{L}_{k}=m_{k}<\infty$, where $\mathcal{L}_{k}$ is the subspace consisting of eigen and associated vectors corresponding to the eigenvalue $\mu_{k}$. These points are $m_{k}$-multiple zeros of the function $\operatorname{det}(I-A(\mu))$.

Now let $H$ be a normal compact operator in $\mathfrak{G}$. Suppose that in a certain sector $\Lambda$ in the complex plane the characteristic numbers of operator $H, \mu_{k}(H)=\lambda_{k}^{-1}(H)$, are concentrated asymptotically along a ray $\gamma \in \Lambda$. We assume that $\gamma$ is the positive semiaxis; then our hypothesis means that not more than a finite number of $\left\{\mu_{k}(H)\right\}$ are contained in the domain $\Lambda_{\alpha_{o}} \backslash \Lambda_{\alpha}$ for some $\alpha_{0}$ and any $\alpha>\alpha_{0}$.

With operator $H$ we connect the operator-valued function

$$
L(\mu)=I-T-A(\mu)-\mu H,
$$

where $I$ is the identity operator, $T \in \sigma_{\infty}, A(\mu)$ is a holomorphic operator-valued function in the sector $\Lambda_{\alpha_{0}}$ with values in $R$ and $\|A(\mu)\| \rightarrow 0$ as $\mu \rightarrow \infty$ in $\Lambda_{\alpha_{0}}$. Suppose also $(I-T)^{-1} \in R$.

Take any $\alpha>\alpha_{0}$ and consider the functions

$$
n_{H}(\alpha, r)=\sum_{\substack{\mu_{k}(H) \leqslant r \\ \mu_{k}(H) \in \Lambda_{\alpha}}} 1 ; \quad n_{L}(\alpha, r)=\sum_{\substack{\mu_{k}(L) \leqslant r \\ \mu_{k}(L) \in \Lambda_{\alpha}}} 1 .
$$

We suppose that a number $\mu_{k}(H)$ or $\mu_{k}(L)$ is repeated in (5.2) $s$ times, if it is an $s$-multiple eigenvalue of $H$ or $L$, respectively.

THEOREM 3. Let $H$ be a compact normal operator and $n_{H}\left(\alpha_{0}, r\right)-n_{H}(\alpha, r) \leqslant C(\alpha)$ for some $\alpha_{0}$ and any $\alpha>\alpha_{0}$. If $L(\mu)$ is defined by (5.1) and $n_{H}\left(\alpha_{0}, r\right) \in C \cap B$, then

$$
n_{H}(\alpha, r) \sim n_{L}(\alpha, r), \quad \alpha>\alpha_{0} \text {. }
$$

Proof. Let $\mathfrak{\mathscr { Q }}=\mathfrak{Q}_{1} \oplus \mathfrak{S}_{2}$ where $\mathfrak{Q}_{1}$ is the subspace consisting of all eigenvalues of the operator $H$ corresponding to $\left\{\mu_{k}(H)\right\} \in \Lambda_{\alpha_{0}}$. Define by $P$ the projector $P \mathfrak{S}_{\mathscr{E}}=\mathfrak{S}_{1}$ and $Q=I-P$. Since $H$ is a normal operator, we have $P Q=Q P=0$. 
Now we represent the operator-valued function $L(\mu)$ in the form

$$
\begin{aligned}
L(\mu) & =I-T-A(\mu)-\mu(P+Q) H \\
& =\left[I-T(Q+P)(I-\mu Q H)^{-1}-A(\mu)(I-\mu Q H)^{-1}\right. \\
& \left.\quad-\mu P H(I-\mu Q H)^{-1}\right](I-\mu Q H) \\
& =\left[I-T P-T Q(I-\mu Q H)^{-1}-A(\mu)(I-\mu Q H)^{-1}-\mu P H\right](I-\mu Q H) .
\end{aligned}
$$

Since $(I-T)^{-1} \in R$, we have $(I-T P)^{-1} \in R$. The operator $Q H$ is a normal operator and $\left\{\mu_{k}(Q H)\right\} \in \Lambda_{\alpha_{0}}$, hence (see [6, Chapter 5, §7])

$$
\left\|(I-\mu Q H)^{-1}\right\| \leqslant \sin ^{-1} \frac{\pi}{2}\left(1 / \alpha_{0}-1 / \alpha\right),
$$

if $\mu \in \Lambda_{\alpha}, \alpha>\alpha_{0}$.

Let $T \in \sigma_{\infty}$; then

$$
\left\|T Q(I-\mu Q H)^{-1}\right\| \rightarrow 0
$$

uniformly as $\mu \rightarrow \infty$ in $\Lambda_{\alpha}$. This assertion is proved in [6, Chapter 5, §7], if $Q=I$ and $H>0$. But absolutely the same method allows us to obtain (4.4). Using (4.3) and (4.4) we represent $L(\mu)$ in the form

$$
L(\mu)=\left(I-T_{1}-A_{1}(\mu)\right)^{-1}\left(I-\mu\left(I-T_{1}-A_{1}(\mu)\right) P H\right)(I-\mu Q H)
$$

where

$$
\begin{gathered}
I-T_{1}-A_{1}(\mu)=\left(I-T P-A_{2}(\mu)\right)^{-1}, \\
A_{2}(\mu)=T Q(I-\mu Q H)^{-1}-A(\mu)(I-\mu Q H)^{-1},
\end{gathered}
$$

$T_{1} \in \sigma_{\infty}$ and $\left\|A_{i}(\mu)\right\| \rightarrow 0, i=1,2$, as $\mu \rightarrow \infty$ in $\Lambda_{\alpha}$.

Define $H_{1}=P H$ and

$$
L_{1}(\mu)=I-\mu\left(I-T_{1}-A_{1}(\mu)\right) H_{1} .
$$

First we consider the case $n_{H}\left(\alpha_{0}, r\right)=n_{H_{1}}(r) \in A_{p}, p<1$. In this case $H_{1} \in \sigma_{1}$ and the functions $\operatorname{det}\left(I-\mu H_{1}\right)$ and $\operatorname{det} L_{1}(\mu)$ are well defined and holomorphic in $\Lambda_{\alpha}$. If $A, B \in \sigma_{1}$, then (see [6, Chapter 4, §3])

$$
\operatorname{det}(I-A)(I-B)^{-1}=\operatorname{det}(I-A) / \operatorname{det}(I-B) \text {. }
$$

Using this formula and (5.6) we obtain

$$
\begin{aligned}
\operatorname{det} L_{1}(\mu) / \operatorname{det}\left(I-\mu H_{1}\right) & =\operatorname{det}(I-\mu K(\mu)), \\
\operatorname{det}\left(I-\mu H_{1}\right) / \operatorname{det} L_{1}(\mu) & =\operatorname{det}(I-\mu K(\mu))^{-1} \\
& =\operatorname{det}\left(I+\mu K(\mu)(I-\mu K(\mu))^{-1}\right)
\end{aligned}
$$

where

$$
K(\mu)=\left(T_{1}+A_{1}(\mu)\right)\left(I-\mu H_{1}\right)^{-1} H_{1} .
$$

Now we recall some properties of $s$-numbers of compact operators (see [6, Chapter 2, §2.3]).

We have

$$
s_{j}(B A) \leqslant\|B\| s_{j}(A), \quad s_{j}(A B) \leqslant\|B\| s_{j}(A),
$$


if $A \in \sigma_{\infty}$ and $B \in R$,

$$
s_{j+\kappa}(B A) \leqslant s_{\kappa+1}(B) s_{j}(A),
$$

if $A \in \sigma_{\infty}, B \in \sigma_{\infty}$ and $j \geqslant+1$.

For $A \in \sigma_{1}$ we also have

$$
\left|\prod_{j=1}^{\infty}\left(1+\lambda_{j}(A)\right)\right| \leqslant \prod_{j=1}^{\infty}\left(1+s_{j}(A)\right) .
$$

Since $H_{1}$ is a normal operator and for any $\beta<\infty$ the set $\left\{\mu_{k}\left(H_{1}\right)\right\} \in \Lambda_{\beta}$ with the exception of a finite number of $\mu_{k}\left(H_{1}\right)$, we obtain

$$
\left\|\left(I-\mu H_{1}\right)^{-1}\right\| \leqslant \sin ^{-1} \frac{\pi}{2}\left(\frac{1}{\alpha}-\frac{1}{\beta}\right) \leqslant \sin ^{-1} \frac{\pi}{4 \alpha}
$$

if $|\mu|>r_{0}$ and $\beta / 2>\alpha>\alpha_{0}$.

Given $\varepsilon>0$, take integer $\kappa$ such that $s_{\kappa+1}\left(T_{1}\right) \leqslant \varepsilon \sin \pi / 4 \alpha$. Then, using (5.10), (5.11) and recalling that $\left\|A_{1}(\mu)\right\| \rightarrow 0$ as $\mu \rightarrow \infty$ in $\Lambda_{\alpha_{0}}$, we obtain from (5.9):

$$
s_{j+\kappa}(K(\mu)) \leqslant 2 \varepsilon s_{j}\left(H_{1}\right), \quad|\mu|>r_{0}, \mu \in \Lambda_{\alpha_{0}}-\Lambda_{\beta} .
$$

Now (5.12) implies

$$
|\operatorname{det}(I-\mu K(\mu))| \leqslant C|\mu|^{\kappa} \prod_{j=1}^{\infty}\left(1+2 \varepsilon|\mu| s_{j}\left(H_{1}\right)\right) .
$$

We claim

$$
\left\|(I-\mu K(\mu))^{-1}\right\| \leqslant C
$$

as $\mu \in \Lambda_{\alpha}-\Lambda_{\beta}, \beta>\alpha>\alpha_{0}$ and $|\mu|>r_{0}$. Once this is known, we obtain from (5.9) and (5.14):

$$
\left|\operatorname{det}(I-\mu K(\mu))^{-1}\right| \leqslant C|\mu|^{\kappa} \prod_{j=1}^{\infty}\left(1+\varepsilon|\mu| s_{j}\left(H_{1}\right)\right) .
$$

Once again as in Lemma 9 (see (3.9) and (3.10)), we have, using our hypothesis $n_{H_{1}}(r) \in A_{p}, p<1$, that

$$
\left|\sum_{j=1}^{\infty} \ln \left(1-\frac{\mu}{\mu_{j}\left(H_{1}\right)}\right)\right| \leqslant \sum_{j=1}^{\infty} \ln \left(1+\frac{|\mu|}{\left|\mu_{j}\left(H_{1}\right)\right|}\right)=O\left(n_{H_{1}}(r)\right) .
$$

Then (5.15) and (5.17) give

$$
\begin{aligned}
\pm \ln \left|\operatorname{det} L_{1}(\mu) / \operatorname{det}\left(I-\mu H_{1}\right)\right| & = \pm \ln |\operatorname{det}(I-\mu K(\mu))| \\
& \leqslant C+r^{\kappa}+\sum_{j=1}^{\infty} \ln \left(1+\varepsilon r s_{j}\left(H_{1}\right)\right) \\
& =C+r^{\kappa}+\sum_{j=1}^{\infty} \ln \left(1+\frac{\varepsilon r}{\left|\mu_{j}\left(H_{1}\right)\right|}\right) \\
& =O\left(n_{H_{1}}(\varepsilon r)\right)=o\left(n_{H_{1}}(r)\right)
\end{aligned}
$$

as $|\mu|=r \rightarrow \infty$ and $\mu \in \Lambda_{\alpha} \backslash \Lambda_{\beta}, \beta>\alpha>\alpha_{0}$. 
The last estimate shows that we can apply Theorem 2 and deduce $n_{L_{1}}(\alpha, r) \sim$ $n_{H_{1}}(r)$. But (5.5) and (5.6) imply that $\left|n_{L}(\alpha, r)-n_{L_{1}}(\alpha, r)\right| \leqslant C$, hence $n_{L_{1}}(\alpha, r) \sim$ $n_{H}(\alpha, r)$ and Theorem 3 follows.

Now prove (5.16). We have from (5.5), (5.6) and (5.9):

$$
\begin{aligned}
I-\mu K(\mu) & =L_{1}(\mu)\left(I-\mu H_{1}\right)^{-1} \\
& =\left(I-T_{1}-A_{1}(\mu)\right)\left(I-T P-A_{2}(\mu)-\mu H_{1}\right)\left(I-\mu H_{1}\right)^{-1} \\
& =\left(I-T_{1}-A_{1}(\mu)\right)\left(I-T P(I-\mu P H)^{-1}-A_{2}(\mu)\left(I-\mu H_{1}\right)^{-1}\right) .
\end{aligned}
$$

In (5.4) replace $Q$ by $P$ and recall (5.13). Then (5.16) follows from (5.19).

We proved Theorem 3 under hypothesis $n_{H_{1}}(r) \in A_{p}, p<1$. If $p>1$, then take integer $l>p$ and consider instead of $L_{1}(\mu)$ the operator-valued function

$$
\begin{aligned}
L_{l}(\mu) & =I-\mu^{l}\left(I-T_{1}-A_{1}(\mu) H_{1}\right)^{l} \\
& =\prod_{k=1}^{l}\left(I-w_{k} \mu\left(I-T_{1}-A_{1}(\mu) H_{1}\right),\right.
\end{aligned}
$$

where $w_{k}^{l}=1, k=1, \ldots, l$. It follows from (5.20) that $\left|n_{L_{1}}(\alpha, r)-n_{L_{l}}(\alpha, r)\right|<C$ if $\alpha<\alpha_{1}=\max \left(\alpha_{0}, l\right)$. The functions $\operatorname{det} L_{l}(\mu)$ and $\operatorname{det}\left(I-\mu^{l} H_{1}^{l}\right)$ are well $\operatorname{defined}$ and holomorphic in $\Lambda_{\alpha_{1}}$. As before, we have to show

$$
\pm \ln \left|\operatorname{det} L_{l}(\mu) / \operatorname{det}\left(I-\mu^{l} H_{1}^{l}\right)\right|=o\left(n_{H_{1}}(r)\right),
$$

as $\mu \rightarrow \infty$ in $\Lambda_{\alpha_{1}} \backslash \Lambda_{\alpha}$ for any $\alpha>\alpha_{1}$, and then to apply Theorem 2. But it is clear that we may obtain (5.21) in the same way as (5.18). This completes the proof of Theorem 3.

COROLlARY. Let $H$ be a compact normal operator, whose characteristic numbers $\mu_{k}(H)$ lie on some rays $\gamma_{1}, \ldots, \gamma_{m}$ in the complex plane and $n_{H}\left(\gamma_{j}, r\right) \in B \cap C$, $j=1, \ldots, m$.

Let $L(\mu)$ be defined by (5.1) and $A(\mu)$ be a holomorphic function in the neighborhood of $\infty$, such that $A(\infty)=0$. Then the eigenvalues of $L(\mu)$ are concentrated asymptotically along the rays $\gamma_{1}, \ldots, \gamma_{m}$ and

$$
n_{H}\left(\gamma_{j}, r\right) \sim n_{L}\left(\gamma_{j}, r\right), \quad j=1, \ldots, m
$$

(we define $n_{L}\left(\gamma_{j}, r\right)$ by (5.2), where $\Lambda_{\alpha}$ is replaced by some small sector $\Lambda$ containing $\left.\gamma_{j}\right)$.

Note 1. Theorem 3 will be valid if we assume in (5.1) (see (5.6))

$$
\left\|A(\mu) H^{-1}\right\|=o(|\mu|), \quad \mu \rightarrow \infty, \mu \in \Lambda_{\alpha_{0}},
$$

instead of $\|A(\mu)\|=o(1)$.

Note 2. If $H>0,\left\|A(\mu) H^{-1}\right\|=O(1)$ as $\mu \rightarrow \infty$ and $n_{H}(r)$ satisfies some tauberian conditions (those tauberian conditions involve $n_{H}(r) \in B \cap C$ ) then the assertion of Theorem 3 was announced in [1]. 


\section{REFERENCES}

1. V. A. Avakjan, Asymptotic distribution of eigenvalues of linear bundle perturbed by analytic operator-valued functions, Functional Anal. Appl. 12 (1978), 66-67. (Russian)

2. M. Sh. Birman and M. Z. Solomyak, Collection, Itogi Nauki i Tekhniki, Mathematicheskii Analiz, no. 14, VINITI, Moscow, 1977, pp. 5-58.

3. M. L. Cartwright, Integral functions, Cambridge Univ. Press, New York, 1956.

4. A. Edrei and W. H. J. Fuchs, On the growth of meromorphic functions with several deficient values, Trans. Amer. Math. Soc. 93 (1959), 292-328.

5. B__ Bounds for the number of deficient values of certain classes of meromorphic functions, Proc. London Math. Soc. 12 (1962), 315-344.

6. I. C. Gohberg and M. G. Krein, Introduction to the theory of linear non-self-adjoint operators, Transl. Math. Monos., vol. 18 , Amer. Math. Soc., Providence, R.I., 1969.

7. W. K. Hayman, Meromorphic functions, Oxford Univ. Press, London and New York, 1964.

8. M. V. Keldysh, Eigenvalues and eigenfunctions of certain classes of non-self-adjoint operators, Dokl. Akad. Nauk. SSSR 77 (1951), 11-14.

9. B. Ja. Levin, Distribution of zeros of entire functions, Transl. Math. Monos., vol. 5 , Amer. Math. Soc., Providence, R.I., 1964.

10. E. C. Titchmarsh, The theory of functions, Oxford Univ. Press, London and New York, 1939.

Department of Mathematics, University of California, Berkeley, California 94720

Current address: Department of Mathematics, Moscow State University, Moscow, 117234, USSR 